\title{
Development of video-assisted breast cancer surgery: Initial experience with a novel method for creating working space without prior liposuction
}

\author{
QIAN-FU WU ${ }^{1}$, YING-HUA YU ${ }^{2 *}$, XIAO ZHU $^{2}$, YING CUI $^{3 *}$, QIN-GUO MO $^{2}$, CHANG-YUAN WEI $^{2}$, \\ XUE-JUAN LIN ${ }^{1}$, XUE-YING LIU ${ }^{1}$, WEI-KANG XIE ${ }^{1}$, SHUI GAN ${ }^{1}$ and WEI LEI ${ }^{1}$ \\ ${ }^{1}$ Department of General Surgery of Guiping People's Hospital, Guiping, Guangxi 537200; ${ }^{2}$ Department of Breast Surgery \\ of Guangxi Cancer Hospital and Affiliated Cancer Hospital of Guangxi Medical University; ${ }^{3}$ Department of \\ Experimental Research, The Affiliated Tumor Hospital of Guangxi Medical University, Nanning, Guangxi 530021, P.R. China
}

Received January 11, 2017; Accepted April 13, 2017

DOI: $10.3892 / \mathrm{mco} .2017 .1279$

\begin{abstract}
Endoscopic techniques are promising in breast surgery. In order to create working space, liposuction is widely used in video-assisted breast surgery (VABS). However, the use of liposuction is likely associated with side effects that may partly limit the application of VABS. Therefore, a new technique of endoscopic axillary lymphadenectomy without prior liposuction was developed by our group. A total of 106 female patients underwent VABS, with special adaptation of the video-assisted surgical procedures previously described. Differing from other endoscopic surgery techniques, our adaptations of VABS included the selection of the working instruments, trocar placement, creation of working space, order of axillary lymph node dissection and method of mastectomy. The operative time was 50-180 min (mean, $85.5 \mathrm{~min}$ ). The intraoperative blood loss ranged from 20 to $100 \mathrm{ml}$ (mean, $48 \mathrm{ml}$ ). The mean lymph node number harvested was 11.5 (range, 6-31). No serious intra- or
\end{abstract}

Correspondence to: Dr Ying-Hua Yu, Department of Breast Surgery of Guangxi Cancer Hospital and Affiliated Cancer Hospital of Guangxi Medical University, $71 \mathrm{He}$ Di Lu, Nanning, Guangxi 530021, P.R. China

E-mail: 13237755008@163.com

Dr Ying Cui, Department of Experimental Research, The Affiliated Tumor Hospital of Guangxi Medical University, $71 \mathrm{He}$ Di Lu, Nanning, Guangxi 530021, P.R. China

E-mail: ycuigx@139.com

Abbreviations: VABS, video-assisted breast surgery; MALND, mastoscopic axillary lymph node dissection; ALND, axillary lymph node dissection

*Contributed equally

Key words: video-assisted breast surgery, liposuction, working space, breast cancer, axillary lymph node dissection postoperative complications were recorded. There was no axillary tumor relapse, trocar site tumor implantation or upper limb edema. Without prior liposuction, our new technique of VABS reduced the blood loss volume, endoscopic surgery time, total volume of drainage fluid and, most importantly, the risk of port-site metastases. This new technique appears to have great clinical potential and good prospects for future endoscopic breast surgery development.

\section{Introduction}

The use of endoscopic surgery for the treatment of breast cancer has been widely investigated by multiple medical centers worldwide. These minimally invasive techniques may be associated with various benefits, such as better cosmetic outcome and improved upper limb function (1-3).

However, despite the advantages of endoscopic breast cancer surgery, it has raised certain concerns regarding additional cost in terms of operative time, equipment and training and, most importantly, the risk of port-site metastases (4-6). Previous studies have confirmed that port-site metastases had developed following endoscopic breast cancer surgery $(7,8)$.

Some of these problems may arise from the associated liposuction, which is commonly used in mastoscopic axillary lymph node dissection (MALND) in order to create working space. Liposuction partly limits the development of video-assisted breast surgery (VABS) and it has been reported that metastatic nodes were found in the filtered liposuction fluid (7). Moreover, liposuction increases the operative time and the working space created is limited.

We hereby describe a novel method for creating endoscopic working space without prior liposuction, in order to overcome the abovementioned limitations and to improve the oncological safety of endoscopic techniques.

\section{Patients and methods}

Patients. Between June, 2014 and October, 2015, a total of 106 female breast cancer patients were treated by endoscopic 
breast cancer surgery at the Department of General Surgery of Guiping People's Hospital (Guangxi, China). The patients' age ranged from 31 to 78 years, with a median age of 48 years. A total of 15, 68 and 23 patients had stage I, II and III disease, respectively; 6 patients with stage III disease were administered neoadjuvant therapy for 2-3 courses. The location of the primary tumor was as follows: Lower inner quadrant, $n=21$; upper inner quadrant, $n=16$; lower outer quadrant, $n=39$; upper outer quadrant, $n=27$; and central area, $n=3$. Written informed consent was obtained from all the patients and the study protocol was approved by the Institutional Ethics Committee of Guiping People's Hospital.

Surgical procedures. VABS was performed as described below. Patient's position. Under general anesthesia, the patient was placed in the supine position with the ipsilateral arm in a $90^{\circ}$ abduction. Securing the patient's forearm to the frame of the bed was not considered necessary.

Trocar placement and establishment of gas-space. Using left VABS as an example, two incisions were performed: The first incision was located at the intersection of the vertical nipple line and the horizontal line at the level of $2 \mathrm{~cm}$ below the inframammary fold (Fig. 1A). This step is important for the removal of the lower part of the mammary gland and axillary lymphatic tissue. The second incision was located in the anterior line of the axilla and parallel to the level of $2 \mathrm{~cm}$ below the inframammary fold (Fig. 1B). A 10-mm trocar was inserted via the first incision. From this trocar, $\mathrm{CO}_{2}$ was infused into the internal space between the posterior breast and pectoralis fascia. The pressure was $\sim 8 \mathrm{mmHg}$. A $30^{\circ}$ $10-\mathrm{mm}$ endoscope was inserted via this first trocar. An additional 5-mm trocar was placed in the second incision, through which a $5 \mathrm{~mm}$ long electrocautery probe was inserted (Fig. 1B). This type of developmental operative approach was different from other operations relying on liposuction to create working space (Fig. 2A and B) (9-12). Additionally, it is also different from a previous study investigating MALND without liposuction (Fig. 2C) (13).

Our endoscopic surgery started with dissection between the posterior breast and pectoralis fascia. Tissue dissection and vessel coagulation were achieved using an electrocautery probe via the second trocar. Due to the expansion of the $\mathrm{CO}_{2}$ gas, dissection was easily performed in the space between the posterior breast and pectoralis fascia (Fig. 1C). This is critical for the generation of working space to enable ALND.

Endoscopic ALND. Following establishment of gas-space, additional skin incisions were placed in the lateral periareolar area. A third 5-mm trocar was inserted through the lateral region of the breast to the space between the posterior breast and pectoralis fascia (Fig. 1D). Endoscopic ALND was then performed via this space.

A $30^{\circ} 10-\mathrm{mm}$ endoscope was introduced through the first 10 -mm trocar to allow viewing the axilla from the floor to the apex, bordered by the latissimus dorsi muscle laterally and the pectoralis major muscle superiorly.

The second and the third 5-mm trocars at the level of $2 \mathrm{~cm}$ below the inframammary fold were the main and supplementary operating positions, respectively. The 5-mm long-arm ultrasonic knife and forceps were placed in these positions to facilitate the performance of complex handling in the axilla under endoscopic view. The adipose tissue and lymph nodes attached to the blood vessels and nerves were dissected with an ultrasonic scalpel. During endoscopic surgery with the ultrasonic scalpel, the blood loss volume and operative time were significantly reduced compared with those with the dipolar dissecting forceps and scissors.

This step described above differs from the most commonly performed MALND, in which lipolysis, balloon expansion and other lifting devices were omitted in endoscopic surgery to help establish a gas space in the axilla $(9,14)$. We were able to establish sufficient working space, including the internal space between the posterior breast and pectoralis fascia and inflation region created by sustained air pressure of $\sim 8 \mathrm{mmHg}$. In cases where dissection of the axillary level II and/or level III lymph nodes was required, we were able to create a suitable endoscopic working space by elevating the lateral part of the pectoralis major muscle with anchored sutures to penetrate the skin and fixed the anchored suture outside the skin with a fixing device (Fig. 3A).

Identification and preservation of important anatomical structures. In principle, the axilla was dissected from the lateral pectoralis major muscle and axillary floor to the apex, until the axillary vein was identified. From there onwards, the operation proceeded laterally and downwards. Strip-like and thick structures, likely to be nerves and blood vessels, were preserved. The dissection around some important anatomical structures is further described below:

Intercostobrachial nerve. The intercostobrachial nerve was the first main structure seen under the endoscope and it was dissected from the lateral pectoralis major muscle. This nerve runs from the serratus anterior and intercostal muscle at the junction of the anterior and lateral side of the chest wall and it looks like a 'crossbeam' in the axillary cavity. Adipose tissue and the lymphatics around the nerve were peeled out using an ultrasonic scalpel (Fig. 3B).

Axillary vein. We were able to identify the middle part of the axillary vein when the endoscope was passed over the intercostobrachial nerve. This vein is located in the anterior-lower side of the intercostobrachial nerve. Once the axillary vein was unmasked, we were able to complete the operation safely.

Space between the major and minor pectoralis muscles. The surgical procedure was directed inwards and upwards to enter the space between the major and minor pectoralis muscles (where the Rotter's lymph node is located). The medial thoracic nerve was seen extending from the middle-upper part of the minor pectoralis muscle to the major pectoralis muscle. Dissection of axillary level II. The lateral thoracic artery and part of the axillary vein were identified when the procedure was advanced medially to dissect the adipose tissue and the lymphatics behind the minor pectoralis muscle. Anchoring sutures were always used to elevate the lateral part of the pectoralis major muscle in order to establish a larger working space when the dissection of these regions and/or axillary level III was performed (Fig. 3A). This procedure must be performed with caution, as the smaller and thinner blood vessel branches are easily cut by the ultrasonic scalpel with ensuing bleeding, while the larger structures are preserved.

Long thoracic nerve. When the procedure was directed medially and downwards, the long thoracic nerve $(2-3 \mathrm{~cm}$ below the intercostobrachial nerve) could only be found at the 

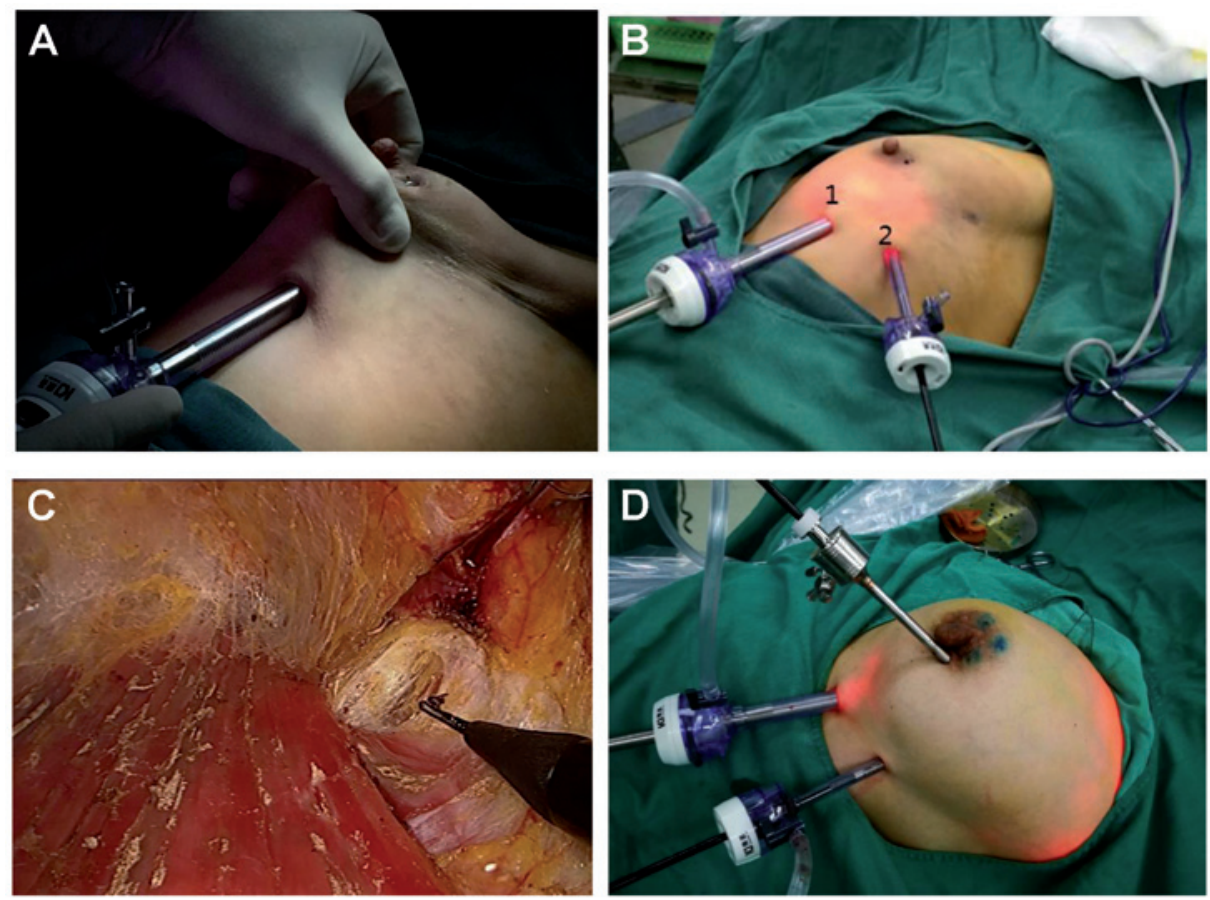

Figure 1. (A) The first position of the trocar was located at the intersection of the vertical line of nipple and the level of $2 \mathrm{~cm}$ below the inframammary fold. (B) Placement of the first two trocars: 1, The first trocar was placed at the intersection of the vertical line of nipple and the level of $2 \mathrm{~cm}$ below the inframammary fold (assistant operating position); 2, the second incision was located at the anterior line of the axilla and parallel to the level of $2 \mathrm{~cm}$ below the inframammary fold (main operating position). (C) The dissection was easily performed in the space between the posterior breast and pectoralis fascia with expansion by $\mathrm{CO}_{2}$ gas. (D) Following establishment of gas space, additional skin incisions were placed in a periareolar lateral location. Via this incision, the third 5-mm trocar was inserted though the lateral region of breast to the internal space between the posterior breast and pectoralis fascia.
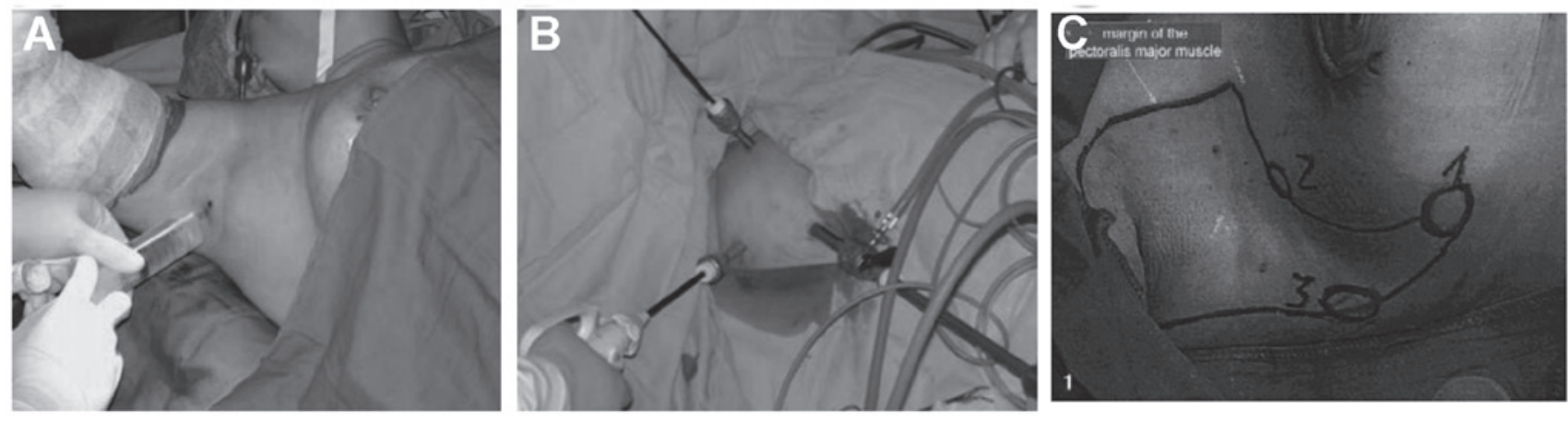

Figure 2. (A) Lipolysis liquid was injected into the axilla; (B) The three trocars were fixed [Chengyu et al (12)]. (C) Position of the trocars for axillary lymphadenectomy. 1, Caudal position of the 10-mm optic trocar; 2, position of the 5-mm trocar at the anterior axillary line; 3, position of the 10-mm trocar at the posterior axillary line. [Kamprath et al (13)].

floor of the axilla, in the deepest layer of the boundaries of the lateral-posterior side of the chest wall. The adipose tissue and lymphatics surrounding the nerve were dissected by the ultrasonic scalpel (Fig. 3C).

Thoracodorsal nerve and vessels and subscapular vessels. The thoracodorsal nerve and vessels and the subscapular vessels were found under the middle part of the axillary vein when the procedure was advanced laterally. Underneath those structures, the subscapular, teres major and latissimus dorsi muscles were identified (Fig. 3C).

Thoracoepigastric vein. It was occasionally seen from the endoscopic view that the thoracoepigastric vein extended from the inferior part of the axillary vein in the lateral part of the minor pectoralis muscle to the anterior chest wall.
Finally, the dissected tissues were temporarily collected at the floor or the apex of the axilla to be removed later. Blood and lymphatic fluid was aspirated using a 5-mm suction device.

The axilla was washed with warm distilled water, which was continuously drained by a suction tube placed in the inferior trocar hole.

Mastectomy. At the extended periareolar skin incision, the dissection between the breast and subcutaneous tissue and along the lateral and medial resection margins was performed using scissors and a scalpel. The blood supply was significantly decreased due to the isolation between the posterior breast and pectoralis fascia, which was performed during the former step. Therefore, excessive bleeding was not expected and haemostasis was achievable through electrical coagulation. 

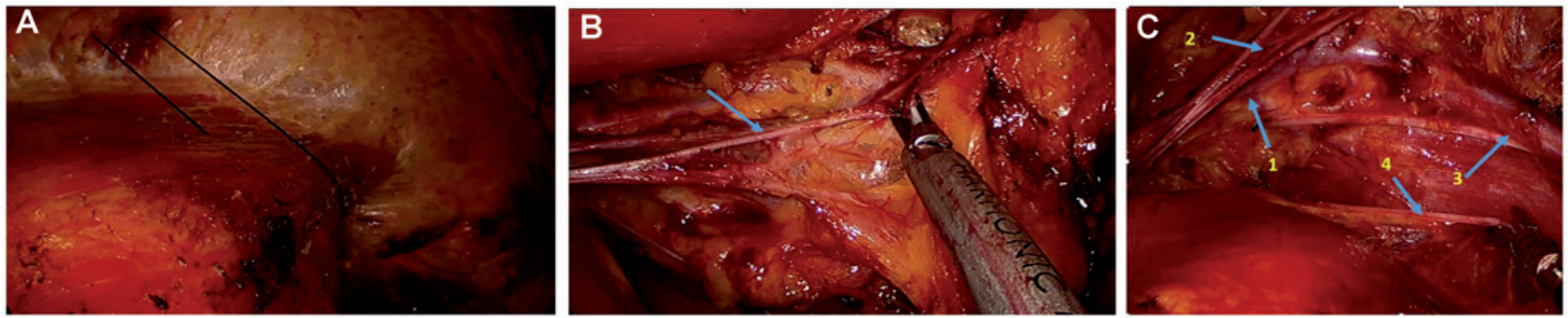

Figure 3. (A) Creation of working space by elevating the lateral part of the pectoralis major muscle with anchoring sutures. (B) Adipose tissues and the lymphatics around the intercostobrachial nerve (blue arrow) were peeled off using an ultrasonic scalpel. (C) Blue arrows indicating important anatomical structures in the axilla: 1 , Axillary vein; 2 , intercostobrachial nerve; 3 , thoracodorsal nerve and vessels; 4 , long thoracic nerve.
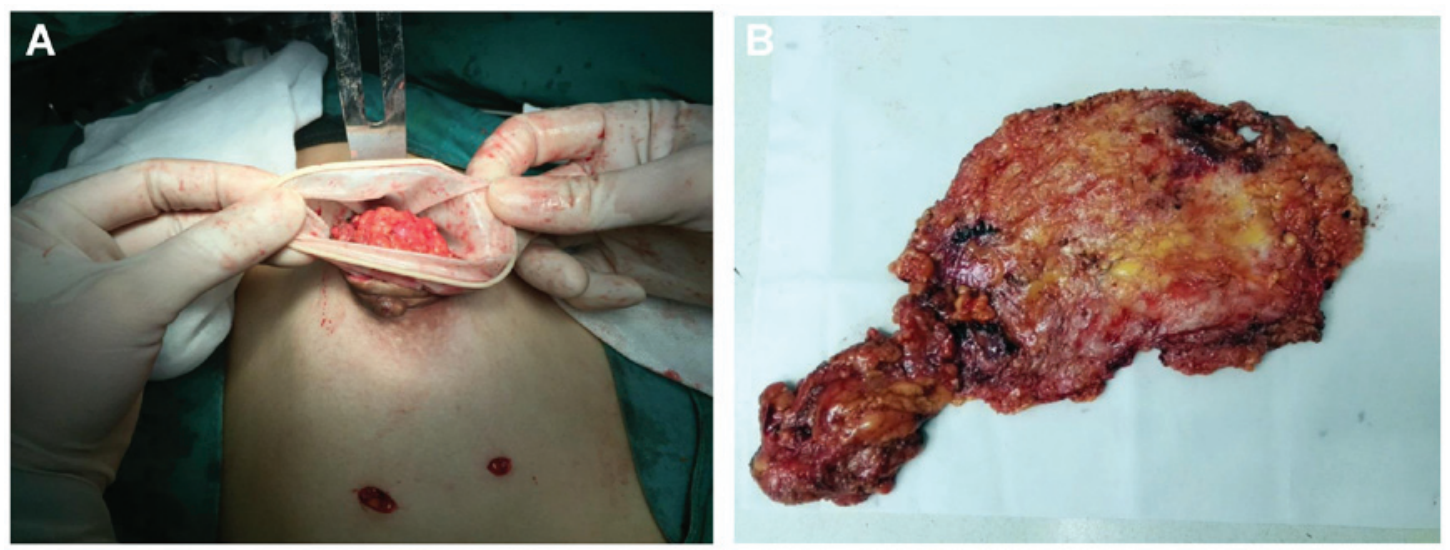

Figure 4. (A) The entire breast specimen and adipose/lymphatic tissues were removed though a 30-mm areolar incision with wound protection. (B) The entire breast specimen and extirpated adipose/lymphatic tissues were completely removed.

Retrieved specimen. The periareolar skin incision through which the trocar was formally placed was extended, and the entire breast specimen was removed, along with extirpated adipose and lymphatic tissues dissected in the axilla, through the extended skin incision and sent for histological assessment (Fig. 4A).

If there was no need to preserve periareolar skin, including the nipple-areola complex, an elliptical incision was performed, incorporating the nipple-areola complex and extending towards the axilla. After that, the entire specimen was retrieved directly through this site.

In order to prevent tumor relapse, the axilla and the space under the skin flap were washed with warm distilled water which was then continuously drained by a suction tube. Finally, the chest and axilla were bandaged.

\section{Results}

Intra- and postoperative parameters. During the entire endoscopic procedure, the blood loss volume was in the range of 20-100 ml, with a mean of $51 \mathrm{ml}$. The mean number of harvested lymph nodes was 12.5 (range, 6-31). A total of 85.8\% (91/106) cases exhibited lymphatic invasion.

The operative time for the entire VABS lasted 55-180 min, with a mean of $85.5 \mathrm{~min}$. The operative time was longer in the earlier procedures, but was shortened once we had become familiarized with the optimal operative pathway and the detailed anatomy of the axilla under the endoscope.
The total volume of drainage fluid in each case ranged from 15 to $250 \mathrm{ml}$. The time to removal of the drainage tubes ranged from 3 to 5 days postoperatively.

There were only 2 trocar holes in the inframammary fold and one incision in the periareolar skin, which were small and easily concealed. Therefore, the majority of the patients were satisfied with the cosmetic results.

No cases were intraoperatively converted to open surgery due to uncontrolled bleeding.

Complications and outcome. There were no serious intraoperative complications, such as injury to the axillary vein, subcutaneous accumulation of fluid, or hematoma. No postoperative complications were reported and postoperative infections were not observed. The shoulder range of motion was not affected in any of the patients. There has been no axillary tumor relapse, trocar site tumor implantation or upper limb edema reported to date.

\section{Discussion}

The rates of complications, including injury to the blood vessels, pain, edema and paraesthesia of the upper limb, continuous lymphedema and limitation of shoulder movement, are comparatively high in conventional ALND $(13,15)$. Furthermore, the incision scar from the anterior chest wall to the axilla may be unsightly and they may also significantly restrict the range of motion of the shoulder joint (16-19). 
Endoscopic techniques have become a promising trend in breast surgery. This technique makes operative anatomy easier with the help of the magnifying function of endoscopic systems, allowing for accurate identification and preservation of the important blood vessels and nerves. In addition, endoscopic techniques are associated with advantages such as satisfactory cosmetic outcome, minimal invasiveness and improved shoulder function. However, in addition to the disadvantages of higher cost due to longer operative time, specialized equipment and training, there are increasing concerns regarding the risk of port-site metastases, which limit the development of endoscopic breast cancer surgery. Some of these issues may be associated with liposuction, which is commonly adopted in MALND to create working space.

In order to create working space, liposuction is commonly used in VABS (2). As a result of this technique, there is no need to extend the breast incision intentionally or unintentionally towards the axilla for performing ALND. Isolation and dissection may then be easily performed with endoscopic instruments $(9,12)$. Liposuction is associated with problems that may limit the use of VABS. There are several disadvantages: First, the amount of fluid injected should be adjusted according to the proportion of fat tissue of the patient. If the liposuction around the axillary vein is not sufficient, the vein cannot be clearly seen and isolation and dissection are difficult to perform with the endoscopic instruments.

Second, the quality of liposuction directly affects the efficiency and efficacy of the endoscopic surgical procedure. When injecting lipolysis liquid and performing lipoaspiration, the surgeon must thoroughly examine all parts of the axilla, particularly the axillary apex and the space between the major and minor pectoralis muscles. These regions are difficult to visualize, which may affect the endoscopic procedure in the axilla and prolong the operative time.

Third, MALND with liposuction is not suitable for obese patients (9). The reason is that, even if the axillary fat is well-aspirated, the created gas space is quite limited, and important structures, such as the axillary vein, lateral thoracic vessels, thoracic epigastric vein and intercostobrachial nerve, are not easily identifiable in the axilla of obese patients.

Fourth, liposuction is a time-consuming procedure, and a sufficient lipolytic effect may require a long time to achieve when MALND is applied (13).

Fifth, if the working trocars and camera are placed too high, some lymphatic tissue may remain in the lower part of the axilla near the insertion site of the trocar.

Finally, the most important limitation may be the risk of port-site tumor implantation. It remains controversial whether endoscopic ALND with liposuction overlooks the general surgical oncological principle of en bloc resection of potentially metastatic lymph nodes. Previous studies confirmed that metastatic nodes were found in the filtered liposuction fluid (7) and histologically confirmed port-site metastases were detected following endoscopic ALND with liposuction $(7,8)$.

To overcome these limitations, a new method for performing video-assisted breast cancer surgery was developed, which may be able to create working space without prior liposuction. In our surgery, the blood loss volume, endoscopic surgery time and total volume of drainage fluid are all obviously more favorable compared with other endoscopic breast surgery studies, as previously reported in a review (20).

In summary, the present study establishes an improved method associated with certain benefits: As regards the choice of endoscope, the $30^{\circ}$ long $( \pm 33 \mathrm{~cm})$ endoscope was selected, as it meets the operative needs, particularly in axillary level II and/or III. Regarding the selection of the surgical instruments, our choices were different from those of other studies regarding the use of short-arm dissecting forceps and scissors $(9,14,21)$. As the distance between the incision port and the dissection working space is somewhat long, long-arm instruments were selected for the endoscopic surgery. The 5-mm long-arm $( \pm 33 \mathrm{~cm})$ dissecting forceps and ultrasonic scalpel are considered suitable for this complex work and they may be more comfortable for the surgeon performing the surgery.

As regards the change of the placement of trocar and creation of working space, in a large number of studies, axillary incisions were the most common point of access to facilitate endoscopic subcutaneous mastectomy, as well as axillary node biopsy (20). In those studies, a total of 3 holes in the axilla were required for MALND (Fig. 2). This placement of the trocars would result in a narrow working space in the axilla. Large or mixed lymph nodes may create more intraoperative difficulties and, due to the narrow space of the axilla, these lymph nodes cannot be easily removed. Therefore, in the majority of MALNDs, enlarged and mixed lymph nodes (diameter, $>1 \mathrm{~cm}$ ) should be excluded. Furthermore, if the working trocars and camera are placed too high, some lymphatic tissue may remain in the lower part of the axilla near the insertion of the trocar.

From our perspective, the choice of trocar placement and the created working space are crucial for creating a successful operative environment. We recommend three incisions as follows: The first incision is located at the intersection of the vertical line of the nipple and the level of $2 \mathrm{~cm}$ below the inframammary fold; the second incision is located at the anterior line of the axilla and parallel to the level of $2 \mathrm{~cm}$ below the inframammary fold; and additional skin incisions are placed in a periareolar lateral location (Fig. 1D). The reason for placing trocars at these sites is that the lower part of the mammary gland and axillary lymphatic tissue cannot be missed during the following procedure, and these positions may help create adequate operative space in the next steps.

As regards working space, our technique did not require other special equipment to create an operative space, such as balloon expansion or a lifting device. The endoscopic procedure started with dissection between the posterior breast and pectoralis fascia. Due to the expansion with $\mathrm{CO}_{2}$ gas, the dissection was simply performed between the posterior breast and pectoralis fascia and sufficient working space was easily created (Fig. 1C). This working space with $8 \mathrm{mmHg}$ $\mathrm{CO}_{2}$ pressure made dissection of the axilla easier. This type of developmental operative approach was different from other operations, which used liposuction to create the initial working space.

As regards the change in the order of endoscopic ALND, creating an internal space between the posterior breast and pectoralis fascia was considered the critical step of endoscopic ALND. Unlike the most common MALND $(9,14)$, lipolysis and balloon expansion or other lifting devices were not required in 
our endoscopic surgery to help establish a gas space in axilla. In addition, the risk of trocar site tumor implantation during liposuction was eliminated.

With sufficient working space, which included the internal space between the posterior breast and pectoralis fascia, and the inflation region created by a sustained air pressure of $\sim 8 \mathrm{mmHg}$, this method is considered more suitable compared with other MALNDs. If the dissection of the axillary level II and/or III is difficult to perform, the lateral part of pectoralis major muscle may be elevated with an anchoring suture that penetrates through the skin and is fixed outwards (Fig. 3A).

With our technique, the axilla was first dissected from the lateral pectoralis major muscle and axillary floor to the apex, until the axillary vein was identified. This surgical order was suitable for identifying the intercostobrachial nerve followed by the axillary vein. Thereafter, the operation proceeded laterally, medially and downwards. This procedure prevents injury to the important structures in the axilla. In addition, some lymphatic tissue remaining in the lower part of the axilla may be completely dissected, unlike with other MALNDs with 3 trocars restricted to the axilla (13).

It is noteworthy that, in several MALND studies, the ultrasonic knife was not recommended, as the short-arm ultrasonic knife is not sufficiently thin or sharp and, therefore, is not considered suitable for severing small and thin strips, or for peeling off the fat and lymphatic tissues around the important nerves and vessels. We consider the 5-mm long-arm ultrasonic knife to be better suited for the complex work in the axilla under endoscopic view. The limited application of ultrasonic scalpel is considered to be largely due to the narrow surgical working space and the type of ultrasonic knife. Although the knife head of the long-arm ultrasonic scalpel is thinner compared with that of the short-arm scalpel, the endoscopic working space created was sufficiently larger to allow use of the long-arm ultrasonic scalpel. During endoscopic surgery with the ultrasonic scalpel, the blood loss volume and the operative time were significantly reduced compared with the bipolar dissecting forceps and scissors.

Regarding the change in the method of the mastectomy, for the majority of VABS procedures, the most commonly employed technique is referred to as the 'subcutaneous tunneling method' (20), which involved creating a number of subcutaneous tunnels using the endoscopic or bladeless trocar. The working planes were created by subcutaneous and sub-mammary elevation, balloon dissection and/or retraction device. Our mastectomy procedure differed significantly from this 'subcutaneous tunnelling method'. Mastectomy is usually performed at the final step. Via the extended periareolar skin incision, scissors and a scalpel were used to perform dissection between the breast and subcutaneous tissue and along the lateral and medial resection margins. After that, the entire breast specimen and extirpated adipose/lymphatic tissues were completely removed through the extended skin incision (Fig. 4B). This procedure is consistent with the general surgical oncological principle and reduces the risk of port-site metastases.

This surgical procedure was familiar to the majority of the surgeons; thus, it was simpler and faster compared with the 'subcutaneous tunneling method'. In addition, the risk of excessive bleeding was reduced due to the blood supply isola- tion, which was performed during the first step of creating a working pace between the posterior breast and the pectoralis fascia. Furthermore, the cosmetic outcome was as satisfactory as that with the 'subcutaneous tunneling method'.

This new endoscopic technique was associated with several advantages: The total operative time, including mastectomy and ALND (mean, $85.5 \mathrm{~min}$ ), was shorter compared with that of other VABS (mean \pm standard deviation, $192.6 \pm 38.5 \mathrm{~min}$ ) (20). The intraoperative blood loss volume (mean, $48 \mathrm{ml}$ ) was also lower compared with other types of VABS (mean, $189 \mathrm{ml}$ ) (20). The total volume of drainage fluid (range, $10-250 \mathrm{ml}$ ) and the time to removal of the drainage tubes (range, 3-5 days) were similar to those recorded for other procedures $(9,21)$. The incidence of intraoperative and postoperative complications was similar to that of other VABS. These advantages were achieved without compromising tumor dissection and operative safety. Most importantly, port-site tumor implantation was not reported, as the method followed the oncological principle of en bloc resection of potentially metastatic lymph nodes.

In the earlier stages, this type of surgery required more time. However, the modified endoscopic technique was optimized after performing 15-30 procedures, which was similar with other endoscopic methods $(9,12)$. Provided the surgeon and the camera operator were experienced, the time was even shorter compared with other types of endoscopic breast surgery.

The limitations of the present study include lack of level I evidence to support this type of VABS, as well as lack of highquality clinical studies, which are required to confirm the oncological success of this type of VABS.

In conclusion, our version of VABS differs from other types of endoscopic surgery regarding the selection of the working instruments, trocar placement, creation of working space, order of ALND and mastectomy method. As it is applied without prior liposuction, this new VABS technique reduces the risk of port-site metastases and it has great clinical potential and good prospects for future development of endoscopic breast surgery.

\section{Acknowledgements}

The authors wish to thank Dr Chiliang Chen and Mrs. Kuili Liang for the helpful discussions. This study was supported by Guangxi Colleges and Universities Science and Technology Research Projects (project number, KY2015LX054) and the Youth Science Foundation of Guangxi Medical University (project number, GXMUYSF201643).

\section{References}

1. Yang W, Sejdinaj F, Shen L, Zhu W, Wang H and Zhang H: Abstract P2-12-05: Trans-peri-areolar breast conservative surgery followed by endoscopic axillary lymph node dissection: A novel surgical option. Cancer Research 76: P2-12-05-P12-12-05, 2016.

2. Wang ZH, Qu X, Teng CS, Ge ZC, Zhang HM, Yuan Z, Gao YG, Lu C, Yu JA and Zhang ZT: Preliminary results for treatment of early stage breast cancer with endoscopic subcutaneous mastectomy combined with endoscopic sentinel lymph node biopsy in China. J Surg Oncol 113: 616-620, 2016.

3. Lai H-W, Chen S-T, Chen D-R, Chen SL, Chang TW, Kuo SJ, Kuo YL and Hung CS: Current Trends in and Indications for Endoscopy-Assisted Breast Surgery for Breast Cancer: Results from a Six-Year Study Conducted by the Taiwan Endoscopic Breast Surgery Cooperative Group. PLoS One 11: e0150310, 2016. 
4. Tamaki Y, Tsukamoto F, Miyoshi Y, Tanji Y, Taguchi T and Noguchi S: Overview: Video-assisted breast surgery. Biomed Pharmacother 56 (Suppl 1): 187s-191s, 2002.

5. Keshtgar MR and Fukuma E: Endoscopic mastectomy: What does the future hold? Wom Health Lond 5: 107-109, 2009.

6. Ingram D; FRCS DIMBMF: Is it time for breast cancer surgeons to embrace endoscopic-assisted mastectomy? ANZ J Surg 78: 837-838, 2008

7. Langer I, Kocher T, Guller U, Torhorst J, Oertli D, Harder F and Zuber M: Long-term outcomes of breast cancer patients after endoscopic axillary lymph node dissection: A prospective analysis of 52 patients. Breast Cancer Res Treat 90: 85-91, 2005.

8. Salvat J, Knopf J-F, Ayoubi J-M, Slamani L, Vincent-Genod A, Guilbert M and Walker D: Endoscopic exploration and lymph node sampling of the axilla. Preliminary findings of a randomized pilot study comparing clinical and anatomo-pathologic results of endoscopic axillary lymph node sampling with traditional surgical treatment. Eur J Obstet Gynecol Reprod Biol 70: $165-173,1996$

9. Chengyu L, Jian Z, Xiaoxin J, Hua L, Qi Y and Chen G: Experience of a large series of mastoscopic axillary lymph node dissection. J Surg Oncol 98: 89-93, 2008.

10. Momeni A, Bannasch H, Torio-Padron N, Borges J and Stark GB: [The application of endoscopy in aesthetic breast surgery]. Handchirurgie, Mikrochirurgie, plastische Chirurgie. Organ Deutschsprachigen Arbeitsgemeinschaft Handchirurgie Organ Deutschsprachigen Arbeitsgemeinschaft Mikrochir Peripheren Nerven Gefasse 38: 144-148, 2006.

11. Ozaki S, Ohara M, Shigematsu H, Sasada T, Emi A, Masumoto N, Kadoya T, Murakami S, Kataoka T, Fujii M, et al: Technical feasibility and cosmetic advantage of hybrid endoscopyassisted breast-conserving surgery for breast cancer patients. J Laparoendosc Adv Surg Tech A 23: 91-99, 2013.

12. Chengyu L, Yongqiao Z, Hua L, Xiaoxin J, Chen G, Jing L and Jian Z: A standardized surgical technique for mastoscopic axillary lymph node dissection. Surg Laparosc Endosc Percutan Tech 15: 153-159, 2005.
13. Kamprath S, Bechler J, Kühne-Heid R, Krause $\mathrm{N}$ and Schneider A: Endoscopic axillary lymphadenectomy without prior liposuction. Development of a technique and initial experience. Surg Endosc 13: 1226-1229, 1999.

14. Lai HW, Wu HS, Chuang KL, Chen DR, Chang TW, Kuo SJ, Chen ST and Kuo YL: Endoscopy-Assisted Total Mastectomy Followed by Immediate Pedicled Transverse Rectus Abdominis Musculocutaneous (TRAM) Flap Reconstruction: Preliminary Results of 48 Patients. Surg Innov 22: 382-389, 2015.

15. Cangiotti L, Poiatti R, Taglietti L, Re P and Carrara B: A miniinvasive technique for axillary lymphadenectomy in early breast cancer: a study of 15 patients. Journal of experimental \& clinical cancer research: CR 18: 295-298, 1999.

16. Ho WS, Ying SY and Chan AC: Endoscopic-assisted subcutaneous mastectomy and axillary dissection with immediate mammary prosthesis reconstruction for early breast cancer. Surg Endosc 16: 302-306, 2002.

17. Brun JL, Rousseau E, Belleannée G, de Mascarel A and Brun G: Axillary lymphadenectomy prepared by fat and lymph node suction in breast cancer. Eur J Surg Oncol 24: 17-20, 1998. (EJSO).

18. Kühn T, Santjohanser C, Koretz K, Böhm W and Kreienberg R: Axilloscopy and endoscopic sentinel node detection in breast cancer patients. Surg Endosc 14: 573-577, 2000.

19. Malur S, Bechler J and Schneider A: Endoscopic axillary lymphadenectomy without prior liposuction in 100 patients with invasive breast cancer. Surg Laparosc Endosc Percutan Tech 11: 38-41, discussion 42, 2001.

20. Leff DR, Vashisht R, Yongue G, Keshtgar M, Yang GZ and Darzi A: Endoscopic breast surgery: Where are we now and what might the future hold for video-assisted breast surgery? Breast Cancer Res Treat 125: 607-625, 2011.

21. Ozaki S and Ohara M: Endoscopy-assisted breast-conserving surgery for breast cancer patients. Gland Surg 3: 94-108, 2014. 